\title{
Maculopatía media paracentral aguda: a propósito de un caso y revisión de la literatura
}

\section{Paracentral acute middle maculopathy: a clinical case report and review of the literature}

\author{
Ana Sanhueza R', Paulina Liberman S. ', Raúl González C.
}

\section{Resumen}

Introducción: la maculopatía media paracentral aguda (PAMM) es una entidad descrita en la tomografía de coherencia óptica de dominio espectral (SD-OCT). Se caracteriza por la presencia de una banda hiperrefringente de localización parafoveal en las capas medias retinales y se manifiesta por la aparición de escotomas paracentrales. En este artículo se presenta un caso clínico con el diagnóstico de PAMM y una breve revisión de la literatura. Métodos: se solicitó consentimiento informado escrito del paciente bajo la aprobación del comité ético-científico de la Facultad de Medicina de la Pontificia Universidad Católica de Chile. La revisión de la literatura se realizó mediante una estrategia de búsqueda que incluyó los términos explicitados en las palabras clave. Resultados: el paciente consulta por un escotoma paracentral superior en el ojo derecho (OD). En la autofluorescencia se observó una lesión hipoautofluorescente parafoveal inferior del OD. La angiografía retinal mostró tenue filtración en la misma zona, sin signos de oclusión arterial. En la SD-OCT del OD, se observó una lesión hiperrefringente parafoveal inferior en las capas medias retinales. El estudio sistémico descartó causas infeccioso-inflamatorias y vasculares. A partir de lo anterior, se realizó el diagnóstico de PAMM. Conclusiones: la presentación clínica y los resultados obtenidos fueron concordantes con lo reportado en la literatura respecto a la PAMM. Actualmente el manejo se basa en buscar y controlar condiciones relacionadas, puesto que no se ha descrito tratamiento específico. En este caso, se descartaron causas secundarias y se decidió la observación clínica.

Palabras clave: PAMM; maculopatía-paracentral; SD-OCT.

\begin{abstract}
Background: Paracentral acute middle maculopathy (PAMM) refers to a recently discovered finding described in the field of spectral-domain optical coherence tomography (SD-OCT). The main hallmark of PAMM is a hyper-reflective band-like lesion involving the middle layers of the retina in a parafoveal position. Typically, patients present with an acute onset of paracentral scotomas. In this article, we report a clinical case of PAMM and a brief review of the relevant literature. Methods: To obtaining clinical information, the requirements included a written informed consent form and the approval of the Ethical-Scientific Committee of Pontificia Universidad Católica de Chile. The literature review was carried out using a search strategy that included the terms specified in the keywords. Results: The patient presented an acute history of a superior paracentral scotoma in the right eye (RE). Fundus autofluorescence uncovered a hypo-autofluorescent area at the inferior fovea in the RE. Fluorescein angiography excluded a retinal artery occlusion and showed a hyperfluorescent capillary leakage at the same location. SD-OCT revealed a hyper-reflective band-like lesion in the middle retinal layers. A complete search for infectious, inflammatory, and vascular causes ruled out secondary conditions. These results were consistent with a diagnosis of PAMM. Conclusions: Clinical presentation and laboratory findings were compatible with the actual evidence concerning PAMM. The current management consists of identification and treatment of related vascular and systemic associated factors because there is no specific treatment for this condition. In this case, a negative study of secondary aetiologies supported the decision of clinical observation.
\end{abstract}

Keywords: PAMM; paracentral-maculopathy; SD-OCT.

Fecha de envío: 12 de julio de 2019 - Fecha de aceptación: 02 de marzo de 2020

\section{Introducción}

La maculopatía media paracentral aguda (PAMM, por sus siglas en inglés "Paracentral Acute Middle Maculopathy") corresponde a una entidad recientemente descrita en la tomografía de coherencia óptica de dominio espectral (SD-OCT) such as spectral domain

(1) Departamento de Oftalmología. Pontificia Universidad Católica de Chile.

Autor de correspondencia: regonzalez@med.puc.cl 
optical coherence tomography (SD-OCT (Sarraf et al., 2013; Rahimy \& Sarraf, 2014). Se caracteriza por la presencia de una banda hiperrefringente a nivel de la capa nuclear interna (INL) de localización parafoveal (Rahimy et al., 2015). Se describe que es secundaria a isquemia en los plexos capilares intermedio y profundo de la retina (Chen et al., 2015).

Clínicamente se manifiesta por la presentación aguda de uno o múltiples escotomas paracentrales, sin otros síntomas oftalmológicos y con hallazgos sutiles o ausentes en el fondo de ojo (Rahimy \& Sarraf, 2014). Los pacientes pueden experimentar déficit visual permanente que es secundario la atrofia de la INL afectada (Rahimy et al., 2015).

En este artículo se presenta el caso de un paciente con diagnóstico de PAMM y una breve revisión de la literatura.

\section{Caso clínico}

Hombre de 27 años, ingeniero, sin antecedentes mórbidos. Consultó de forma ambulatoria en el Servicio de Oftalmología del Centro Médico San Joaquín por un escotoma paracentral superior en el ojo derecho (OD) de inicio agudo y nueve días de evolución. No refería fotopsias, ojo rojo, dolor ocular ni compromiso contralateral. A la anamnesis por sistemas, no presentaba otros síntomas y negaba consumo de tabaco o drogas.

La agudeza visual (AV) sin corrección era 20/20 en ambos ojos. En la biomicroscopía el segmento anterior era normal. En el fondo de ojo se observaron papilas de bordes netos, máculas sanas, retina aplicada y periferia sin lesiones. Al día siguiente fue reevaluado y en el fondo de ojo derecho destacaba una lesión blanquecina paracentral inferior de aspecto no solevantado en relación a una rama arterial secundaria.

Se realizó estudio con autofluorescencia, en la que se observó una lesión hipoautofluorescente en la región parafoveal inferior del OD (Figura 1). En la SD-OCT del mismo ojo, se observó una lesión hiperrefringente en placa de las capas medias de la retina y de ubicación parafoveal inferior (grosor central foveal: $288 \mu \mathrm{m}$ ) (Figura 2). En la angiografía retinal se observó tenue filtración capilar en la región inferior de la fóvea (Figura 3) sin signos de oclusión arterial ni compromiso en la perfusión en OD. Los exámenes del Ol fueron normales.

Se descartaron causas infeccioso-inflamatorias y vasculares con RPR, FTA-ABS, anticuerpos anti-Bartonella, anticuerpos anti-Toxoplasma, anticuerpos anti-DNA, ANA, ANCA, complemento C3-C4, perfil de trombofilia (anticoagulante lúpico, anticuerpos anticardiolipina, anticuerpos anti- $\beta 2$ Glicoproteína I, Factor V Leiden, Protrombina 20210, antitrombina, proteínas Cy S), hemograma, perfil metabólico, ecografía doppler carotídea, ecografía cardíaca, holter de arritmias y RNM de cerebro. Todo el estudio fue negativo.
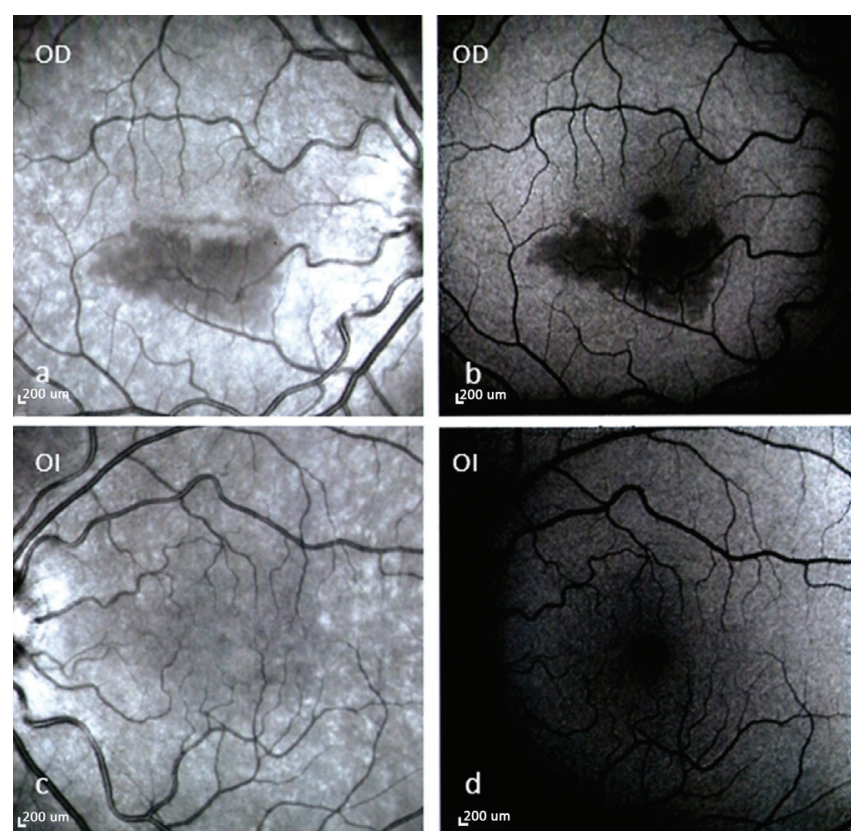

Figura 1: Autofluorescencia $(a, c)$. Se observa lesión hipoautofluorescente de bordes netos y localización parafoveal inferior en OD. Retinografía aneritra (b,d). Se observa área con mayor diferencia de contraste en región parafoveal inferior del OD, cuyas dimensiones fueron $4,86 \mathrm{~mm}$ de largo, 2,36 $\mathrm{mm}$ de ancho y un área aproximada de 7,33 $\mathrm{mm} 2$. OD: Ojo derecho. Ol: Ojo izquierdo. mm: milímetro. Autofluorescencia y retinografía aneritra obtenidas con Equipo SPECTRALIS ${ }^{\oplus}$ HRA+OCT (Heidelberg Engineering $\odot$ ).
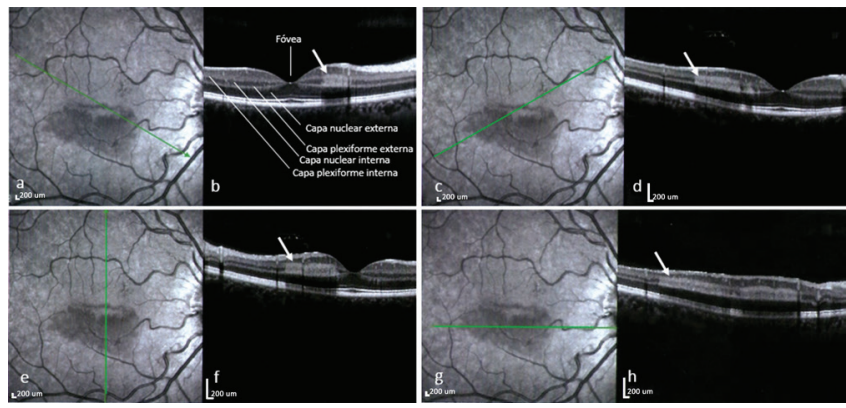

Figura 2: Autofluorescencia (a, c, e, g) y sección correspondiente de la SD-OCT de mácula ( $b, d, f, h)$ del ojo derecho. Se observan lesiones hiperrefringentes en las capas medias retinales (flecha) de localización parafoveal inferior, cuyas dimensiones son 1,72 $\mathrm{mm}$ de ancho (figura f) y 4,79 $\mathrm{mm}$ de largo (figura h). mm: milímetro. Imágenes obtenidas con Equipo SPECTRALIS ${ }^{\oplus}$ $\mathrm{HRA}+\mathrm{OCT}$ (Heidelberg Engineering $\odot$ ). 

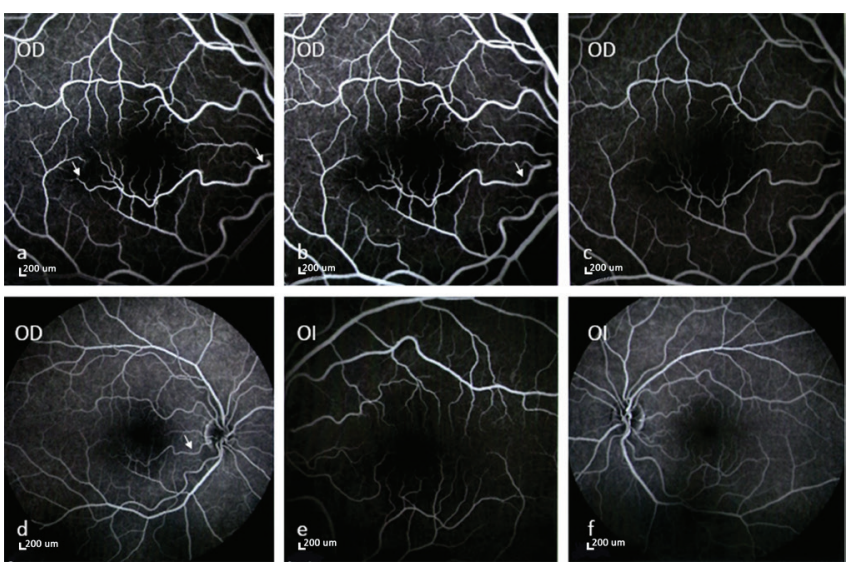

Figura 3: Fases tempranas de angiografía retinal ( $a, b$ de OD y e de Ol). Fases tardías de angiografía retinal: (c, d de OD y f de Ol). Se observa filtración capilar en región parafoveal inferior de OD, secundario al compromiso de la arteria cilioretinal (flecha), sin imágenes sugerentes de oclusión arterial. Sin hallazgos en OI. OD: Ojo derecho. OI: Ojo izquierdo. Imágenes obtenidas con Equipo SPECTRALIS ${ }^{\circledast}$ HRA+OCT (Heidelberg Engineering $\odot$ ).
A partir de lo anterior y de los hallazgos obtenidos en la SD-OCT, se realizó el diagnóstico de PAMM. Dado que no se identificaron causas secundarias, se decidió la observación clínica. Tras cuatro meses desde el inicio del cuadro clínico, el paciente persistía con el escotoma paracentral superior que se evidencia en el campo visual computado. En la SD-OCT se observa atrofia de la INL e hiperrefringencia de las capas medias retinales en región parafoveal inferior de OD (Figura 4).

\section{Discusión}

La PAMM se describió inicialmente en el año 2013 como una variante de la neuroretinopatía macular aguda (AMN). El término se acuñó descriptivamente debido a la presentación aguda de un escotoma paracentral, asociado a una lesión parafoveal hiperrefringente en la SD-OCT (Sarraf et al., 2013). Esta lesión se localiza en las capas medias de la retina. Compromete específicamente la INL y zona de unión entre la capa plexiforme externa y la INL, sin afectar las capas retinales externas (Sarraf et al., 2013; Rahimy et al., 2015).
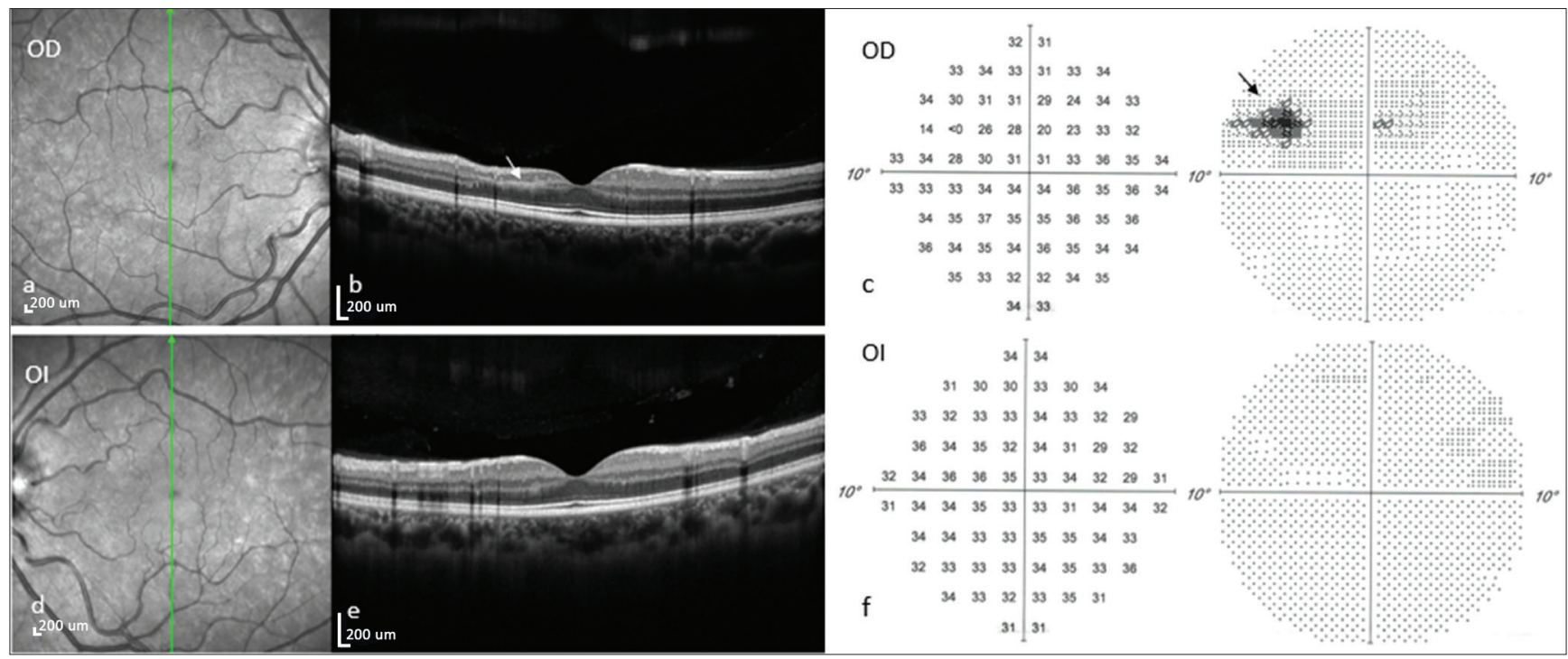

Figura 4: Autofluorescencia ( $a$, d) y sección correspondiente de la SD-OCT de mácula (b, e) a los 4 meses de evolución del cuadro clínico. Se observa atrofia de la INL e hiperrefringencia de las capas medias retinales en región parafoveal inferior de OD (flecha blanca). Sin lesiones en OI. Campo visual 10-2 (c,f) evidencia escotoma paracentral superior en el OD (flecha negra). Sin hallazgos patológicos en el OI. INL: Capa nuclear interna. OD: Ojo derecho. OI: Ojo izquierdo. Autofluorescencia y SD-OCT obtenidas con Equipo SPECTRALIS ${ }^{\circledR}$ HRA+OCT (Heidelberg Engineering $\odot$ ). Campo visual obtenido con Humphrey Field Analizer 3 (Zeiss@).

A diferencia de la PAMM, las lesiones en la AMN se presentan con bandas de atenuación entre la capa plexiforme externa y la capa nuclear externa, lo que se asocia a disrupción macular externa y pérdida del elipsoide (Rahimy et al., 2015; Bhavsar et al., 2016). Lo anterior produce secundariamente un adelgazamiento de la capa nuclear externa (Sarraf et al., 2013; Bhavsar et al., 2016).

En cuanto a la fisiopatología de la PAMM, mediante la angiografía OCT (OCTA) se ha obtenido evidencia de que ésta es secundaria a la isquemia producida en los plexos capilares intermedio y profundo de la retina (Chen et al., 2015; Nemiroff et al., 2016), los cuales están respectivamente localizados en relación a las regiones interna y externa de la INL (Chan et al., 2012; Tan et al., 2012; Sridhar et al., 2015). La localización anatómica de la alteración vascular ha sido difícil de demostrar por angiografía convencional (Chen et al., 2015; Rahimy et al., 2015). No obstante, la OCTA permite obtener imágenes de alta resolución de la vasculatura retinal según profundidad (Rahimy et al., 2015). Con esta nueva técnica 
ha sido posible co-localizar las alteraciones en el flujo de los plexos capilares intermedio y profundo, con las zonas donde se observan las lesiones sugerentes de PAMM en la SD-OCT (Christenbury et al., 2015; Sridhar et al., 2015; Nemiroff et al., 2016).

Si bien la PAMM corresponde a una anormalidad en la SD-OCT, ésta puede indicar una condición subyacente. En la literatura se reporta que la PAMM se asocia a patologías vasculares retinales o sistémicas. Hallazgos característicos de PAMM se han encontrado en retinopatía diabética, oclusión arterial retinal, oclusión de vena central de la retina, retinopatía de células falciformes y retinopatía de Purtscher (Rahimy et al., 2015; Chen et al., 2015; Yu et al., 2015; Ilginis et al., 2015). En series de casos, la PAMM se ha asociado a retinopatía hipertensiva, compresión orbitaria, migraña, hipovolemia y al uso de sustancias como cafeína, anfetaminas, vasopresores o anticonceptivos orales (Sarraf et al., 2013; Chen et al., 2015). Recientemente se ha reportado su asociación con hipertensión endocraneana (Denny et al., 2019) y síndrome antifosfolípidos (Trese et al., 2017). Por otro lado, la PAMM puede ser una condición idiopática y estar presente en pacientes sin comorbilidades (Chen $\& H u, 2017)$. Sin embargo, frente a hallazgos sugerentes de ésta en la SD-OCT, es necesario un estudio meticuloso para descartar factores vasculares sistémicos o retinales (Rahimy et al., 2015).

La PAMM se ha descrito como un hallazgo característico en la SDOCT y no se ha definido como una enfermedad propiamente tal. Sin embargo, puede manifestarse clínicamente por la aparición aguda de escotomas paracentrales en uno o ambos ojos (Sarraf et al., 2013; Rahimy \& Sarraf, 2014). Éstos aparecen como visión borrosa central o“manchas oscuras"fijas (Sarraf et al., 2013; Rahimy \& Sarraf, 2014). La AV puede estar levemente comprometida, pero en la mayoría de los casos no está afectada (Rahimy \& Sarraf, 2014). El fondo de ojo y la retinografía pueden ser completamente normales, aunque en algunos casos existe la presencia de lesiones que pueden ser confundidas con exudados algodonosos (Rahimy \& Sarraf, 2014; Rahimy et al., 2015). A diferencia de éstos, las lesiones asociadas a la PAMM son más profundas en la retina, de contornos menos definidos, apariencia blanquecina-grisácea u opaca y no siguen la distribución de la capa de fibras nerviosas (Rahimy \& Sarraf, 2014; Rahimy et al., 2015). En cuanto a la evolución, los escotomas pueden ser transitorios y presentar mejoría en grado variable, o bien, puede haber un déficit permanente secundario a la atrofia de la INL afectada (Rahimy et al., 2015), tal como en el caso expuesto. A largo plazo, el fondo de ojo es normal o se puede observar un oscurecimiento tenue en la zona afectada (Yu et al., 2014).

Debido a las manifestaciones sutiles o ausentes de la PAMM en el examen de fondo de ojo por biomicroscopía, es necesario contar con métodos diagnósticos complementarios. La retinografía permite obtener imágenes de mayor resolución de la retina, donde es posible observar las lesiones previamente descritas (Rahimy \& Sarraf, 2014). La presencia de áreas hipoautofluorescentes puede ser un hallazgo en la autofluorescencia (Shah et al., 2019). Si bien la angiografía retinal contribuye a descartar la presencia de oclusión arterial retinal (Rahimy et al., 2015), no necesariamente existe correlación anatómica entre las lesiones sugerentes de PAMM en la SD-OCT y las imágenes obtenidas por angiografía. Dicha técnica no es óptima para visualizar el plexo radial peripapilar (Spaide et al., 2015) ni los plexos capilares intermedio y profundo de la retina (Mendis et al., 2010).

En los últimos años se ha producido un aumento progresivo del conocimiento acerca de la PAMM y las alteraciones vasculares asociadas. En consecuencia, es probable que esta condición sea más común de lo que se piensa y por lo tanto, se debiera considerar en aquellos casos con historia sugerente y sin diagnóstico claro. A pesar de lo anterior, no se ha descrito tratamiento específico para ella y por el momento el manejo se basa en buscar y controlar condiciones vasculares que pudieran estar relacionadas (Rahimy et al., 2015).

\section{Fuentes de financiamiento}

Se declara no recibir apoyo financiero.

\section{Contribuciones y conflictos declarados por los autores}

Los autores no declaran conflictos de intereses.

Ana Sanhueza: Concepción y diseño del trabajo, revisión de la literatura, redacción del manuscrito y confección de imágenes, revisión crítica del manuscrito.

Paulina Liberman: Concepción y diseño del trabajo, recolección de datos del caso clínico, revisión crítica del manuscrito, asesoría técnica, aprobación de su versión final.

Raúl González: Aporte del caso clínico, concepción del trabajo, recolección de datos, asesoría técnica, revisión crítica del manuscrito, aprobación de su versión final.

\section{Referencias}

Bhavsar K, Lin S, Rahimy E, Joseph A, Freund K, Sarraf D \& Cunningham E. (2016). Acute macular neuroretinopathy: A comprehensive review of the literature. Survey of Ophthalmology 61, 538-565.

Chan G, Balaratnasingam C, Yu P, Morgan W, McAllister I, Cringle S \&Yu DY. (2012). Quantitative Morphometry of Perifoveal Capillary Networks in the Human Retina. Investigative Ophthalmology \& Visual Science: IOVS 53, 5502-5514. 
Chen X, Rahimy E, Sergott R, Nunes R, Souza E, Choudhry N, Cutler N, Houston S, Munk M, Fawzi A, Mehta S, Hubschman J, Ho A \& Sarraf D. (2015). Spectrum of Retinal Vascular Diseases Associated With Paracentral Acute Middle Maculopathy. American Journal of Ophthalmology 160, 26-34.

Chen Y \& Hu Y. (2017). The optical imaging of idiopathic paracentral acute middle maculopathy in a Chinese young man and review of the literature. Photodiagnosis and Photodynamic Therapy 19, 383-387.

Christenbury J, Klufas M, Sauer T \& SarrafD. (2015). OCT Angiography of Paracentral Acute Middle Maculopathy Associated With Central Retinal Artery Occlusion and Deep Capillary Ischemia. Ophthalmic Surgery Lasers Imaging Retina 46, 579-581.

Denny M, Kalevar A, Chen J \& Johnson R. (2019). Paracentral acute middle maculopathy associated with idiopathic intracranial hypertension. Retinal Cases \& Brief Reports (Volume Publish Ahead of Print).

Ilginis T, Keane PA \& Tufail A (2015). Paracentral acute middle maculopathy in sickle cell disease. JAMA Ophthalmology 133, 614-616.

Mendis K, Balaratnasingam C, Yu P, Barry C, McAllister I, Cringle $S \&$ Yu D. (2010). Correlation of Histologic and Clinical Images to Determine the Diagnostic Value of Fluorescein Angiography for Studying Retinal Capillary Detail. Investigative Opthalmology \& Visual Science: IOVS 51, 5864-5869.

Nemiroff J, Kuehlewein L, Rahimy E, Tsui I, Doshi R, Gaudric A, Gorin M, Sadda S \& Sarraf D. (2016). Assessing Deep Retinal Capillary Ischemia in Paracentral Acute Middle Maculopathy by Optical Coherence Tomography Angiography. American Journal of Ophthalmology 162, 121-132.

Rahimy E, Sarraf D, Dollin M, Pitcher J \& Ho A. (2014). Paracentral acute middle maculopathy in nonischemic central retinal vein occlusion. American Journal of Ophthalmology 158, 372-380.

Sridhar J, Shahlaee A, Rahimy E, Hong B, Khan M, Maguire J, Dunn J, Mehta S \& Ho A. (2015). Optical Coherence Tomography Angiography and en Face Optical Coherence Tomography Features of Paracentral Acute Middle Maculopathy. American Journal of Ophthalmology 160, 1259-1268.
Tan P, Yu PK, Balaratnasingam C, Cringle S, Morgan W, McAllister I \& Yu DY. (2012). Quantitative Confocal Imaging of the Retinal Microvasculature in the Human Retina. Investigative Ophthalmology \& Visual Science: IOVS 53, 5728-5736.

Trese M, Thanos A, Yonekawa Y \& Randhawa S. (2017). Optical Coherence Tomography Angiography of Paracentral Acute Middle Maculopathy Associated With Primary Antiphospholipid Syndrome. Ophthalmic Surgery Lasers Imaging Retina 48, 175-178.

Rahimy E \& Sarraf D. (2014). Paracentral acute middle maculopathy spectral-domain optical coherence tomography feature of deep capillary ischemia. Current Opinion in Ophthalmology 25, 207-212.

Rahimy E, Kuehlewein L, Sadda S \& Sarraf D. (2015). Paracentral Acute Middle Maculopathy: What We Knew Then and What We Know Now. Retina 35, 1921-1930.

Sarraf D, Rahimy E, Fawzi A, Sohn E, Barbazetto I, Zacks D, Mittra R, Klancnik JM Jr, Mrejen S, Goldberg N, Beardsley R, Sorenson J \& Freund KB. (2013). Paracentral acute middle maculopathy: a new variant of acute macular neuroretinopathy associated with retinal capillary ischemia. JAMA Ophthalmology 131, 1275-1287.

Shah A, Rishi P, Chendilnathan C \& Kumari S. (2019). OCT angiography features of paracentral acute middle maculopathy. Indian journal of ophthalmology 67, 417-419.

Spaide R, Klancnik J \& Cooney M. (2015). Retinal Vascular Layers Imaged by Fluorescein Angiography and Optical Coherence Tomography Angiography. JAMA Ophthalmology 133, 45-50.

Yu S, Wang F, Pang C, Yannuzzi L \& Freund K. (2014). Multimodal imaging findings in retinal deep capillary ischemia. Retina 34, 636-646.

Yu S, Pang C, Gong Y, Freund K, Yannuzzi L, Rahimy E, Lujan B, Tabandeh H, Cooney MJ \& Sarraf D. (2015). The spectrum of superficial and deep capillary ischemia in retinal artery occlusion. American Journal of Ophthalmology 159, 53-63. 